THINK TWICE BEFORE YOU START INOTROPIC TREATMENT FOR CIRCULATORY SUPPORT...ARE WE DOING GOOD OR BAD?

I.M. Hirfanoglu', E. Ergenekon ${ }^{2}$, O. Turan², S. Beken², C. Turkyilmaz ${ }^{2}$, E. Onal ${ }^{2}$, E. Koc ${ }^{2}$, Y. Atalay ${ }^{2}$

${ }^{1}$ Pediatrics, Gazi University, ${ }^{2}$ Pediatrics, Gazi University, Besevler, Ankara, Turkey

Objective: The objective of this study was to determine microcirculation by "side stream dark field (SDF) imaging" in neonates receiving inotropic support for circulatory failure.

Method: Neonates needing circulatory support were included.Macrocirculatory(blood pressure, heart rate,capillary refilling times) and microcirculatory parameters(Total vessel density(TVD), perfused vessel density(PVD), microvascular flow index(mfi) and Debacker index) were measured before and after inotropes.

Results: 33 patients were included;GA:32.7 \pm 4.4 weeks;BW:1900 \pm 942 g.59\% were premature,21\% had sepsis, $20 \%$ had other diseases. Seventynine $\%$ received dopamine only, $18 \%$ received dopamine+dobutamine, $6 \%$ received dopamine,dobutamine and adrenaline and one patient received hydrocortisone additionally. Circulatory findings are shown in Table

\begin{tabular}{|l|l|l|l|}
\hline & $\begin{array}{l}\text { Before } \\
\text { inotrope } \\
\text { Mean } \pm \text { SD } \\
\text { (median) }\end{array}$ & $\begin{array}{l}\text { After inotrope } \\
\text { Mean } \pm \text { SD } \\
\text { (median) }\end{array}$ & $p$ \\
\hline $\begin{array}{l}\text { Mean } \\
\text { blood } \\
\text { pressure } \\
\text { (mmHg) }\end{array}$ & $29,4 \pm 7,5(30)$ & $35,1 \pm 9,6(35)$ & $<0,001$ \\
\hline $\begin{array}{l}\text { Capillary } \\
\text { refilling } \\
\text { time (s) }\end{array}$ & $3,7 \pm 0,5(3.5)$ & $2,8 \pm 0,9(2.5)$ & $<0,001$ \\
\hline $\begin{array}{l}\text { mfi(small } \\
\text { vessels) }\end{array}$ & $2,6 \pm 0,6(2.7)$ & $2,1 \pm 0,5(2.2)$ & $<0,001$ \\
\hline $\begin{array}{l}\text { mfi(all } \\
\text { vessels) }\end{array}$ & $2,2 \pm 0,4(2.1)$ & $2,6 \pm 0,7(2.7)$ & $<0,001$ \\
\hline $\begin{array}{l}\text { Debacker } \\
\text { index }\end{array}$ & $6,9 \pm 0,9(7)$ & $7,5 \pm 1,4(8)$ & $<0,001$ \\
\hline $\begin{array}{l}\text { PVD } \\
\text { (small } \\
\text { vessels) }\end{array}$ & $8,6 \pm 2,6(7.9)$ & $8,8 \pm 2,3(8.9)$ & 0,82 \\
\hline $\begin{array}{l}\text { TVD } \\
\text { (small } \\
\text { vessels) }\end{array}$ & $10,4 \pm 3,2(10.8)$ & $11,2 \pm 3,2(11.8)$ & $\begin{array}{l}11,2 \pm \\
3,2(11.8)\end{array}$ \\
\hline $\begin{array}{l}\text { PVD(all } \\
\text { vessels) }\end{array}$ & $10,5 \pm 3,3(10.9)$ & $11,3 \pm 3,3(12)$ & 0,03 \\
\hline $\begin{array}{l}\text { TVD(all } \\
\text { vessels) }\end{array}$ & $11,5 \pm 2,4(11.3)$ & $12,0 \pm 2,7(12.3)$ & 0,21 \\
\hline
\end{tabular}

[Macro and microcirculation data]

Discussion: While there was significantimprovement in macrocirculation and increase in mfi values in all vessels there was decrease in the mfi values in the vessels with diameter under 25 micrometer,following inotropic treatment. This might lead to tissue hypoxia. While correcting macrocirculation with inotrops we might be disrupting microcirculation at cellular level. Nevertheless for further studies on effects of inotrops for circulatory support are needed.

102

\section{A COMPARISON OF CONTINUOUS MULTI- CHANNEL EEG WITH AEEG FOR THE PREDICTION OF OUTCOME AT 24 MONTHS IN HYPOXIC-ISCHAEMIC ENCEPHALOPATHY}

D.M. Murray ${ }^{1}$, E. Lowe ${ }^{1}$, D. O’Rourke ${ }^{1}$, D. Azzopardi², L. Hellstrom Westas ${ }^{3}$, G.B. Boylan ${ }^{1}$

${ }^{1}$ Paediatrics and Child Health, Univerisity College Cork, Cork, Ireland, ${ }^{2}$ Imperial College London, London, UK, ${ }^{3}$ Lund University, Lund, Sweden

Background: Accurate prediction of outcome in hypoxic-ischaemic encephalopathy (HIE) remains 